\title{
Criticality of Large Delay Tolerant Networks via Directed Continuum Percolation in Space-Time
}

\author{
Esa Hyytiä and Jörg Ott \\ Department of Communications and Networking \\ Aalto University, Finland
}

\begin{abstract}
We study delay tolerant networking (DTN) and in particular, its capacity to store, carry and forward messages so that the messages eventually reach their final destination(s). We approach this broad question in the framework of percolation theory. To this end, we assume an elementary mobility model, where nodes arrive to an infinite plane according to a Poisson point process, move a certain distance $\ell$, and then depart. In this setting, we characterize the mean density of nodes required to support DTN style networking. In particular, under the given assumptions, we show that DTN communication is feasible when the mean node degree $\nu$ is greater than $4 \cdot \eta_{\mathrm{c}}(\gamma)$, where parameter $\gamma=\ell / d$ is the ratio of the distance $\ell$ to the transmission range $\mathrm{d}$, and $\eta_{\mathrm{c}}(\gamma)$ is the critical reduced number density of tilted cylinders in a directed continuum percolation model. By means of Monte Carlo simulations, we give numerical values for $\eta_{\mathrm{c}}(\gamma)$. The asymptotic behavior of $\eta_{\mathrm{c}}(\gamma)$ when $\gamma$ tends to $\infty$ is also derived from a fluid flow analysis.
\end{abstract}

Index Terms-DTN, capacity, percolation, criticality, mobility

\section{INTRODUCTION}

Delay-tolerant networking (DTN) is a last-resort networking paradigm for mobile nodes when direct and multi-hop connections are infeasible, i.e., the network is not connected and nodes have to carry the messages to the next node (store-carryforward). We study the capacity of DTN to deliver a message to its destination(s) in the framework of the percolation theory. To this end, we assume an elementary mobility model where nodes arrive according to a Poisson point process on a plane, move a certain distance $\ell$, and then depart. Nodes perform epidemic routing, i.e., whenever two nodes meet, all messages are exchanged. We are interested in finding the circumstances under which the lifetime of a message becomes infinite (with some positive probability) so that the message could reach a recipient located at an arbitrary distance from the source. We obtain a fundamental criticality condition that characterizes the sufficient mean density of nodes to this end. The criticality condition takes form $\nu>\nu_{c}(\gamma)=4 \eta_{c}(\gamma)$, where $\nu$ denotes the mean node degree (number of neighbors), $\gamma$ is the ratio of $\ell$ to the transmission range $d$, and $\eta_{c}(\gamma)$ is an unknown function, which we determine in this paper.

For a mobile DTN, we find that $\nu_{c}(\gamma) \leq 1.52$ for all $\gamma$, while only at $\nu \approx 4.51$ a (non-DTN) wireless ad-hoc network percolates and a gigantic connected cluster emerges [1]. In other words, DTN communication is possible over a very sparse network provided that the network's topology changes in time. A convenient characterization in fact is to say that DTN is a network that is super-critical in space-time. In practice, DTN is often sub-critical at a random time instant and the messages find their way to the destination through the store-carry-forward routing in space-time.

This work is motivated by different opportunistic networking schemes. One such scheme is Floating Content, where nodes replicate messages only within the area where each message is deemed relevant. A criticality condition, characterizing the circumstances under which the lifetime of the message can be expected to be long, was established in [2] under the assumptions of mobile nodes and point contacts (i.e., the transmission range is small compared to the dimension of the area). Similarly, Beachnet [3] the aim is to produce quasi-periodic "information waves" carried by an underlying field of immobile nodes (say, devices on a beach). Beachnet is designed to offer regular content dissemination when nodes are (mostly) stationary and their collective cooperation, based on simple local transmission rules, is sought. Similar concepts for one-to-many content dissemination include hovering information [4] and ad-hoc podcasting [5], but our model naturally covers the special case of one-to-one messaging. These types of DTN schemes introduce many interesting problems. Perhaps the most fundamental question is whether a network can transport messages to their intended destinations or not, and this is also our focus in this paper.

\section{A. Background and related work}

We assume epidemic routing which was proposed by Vahdat and Becker in [6]. The operational principle is very simple: whenever two nodes meet they exchange the messages only one of them has. The performance analysis of the epidemic routing often assumes exponentially distributed (i.i.d.) intermeeting times leading to Markovian models [7]. As the size of the network grows, solving Markov chains becomes infeasible, and Zhang et al., in [8] obtained ODEs as a fluid limit of such Markovian models. In above work, the spatial dimension has been abstracted away.

Jacquet et al. [9] consider the propagation speed of the information in DTN for a fixed set of nodes moving in a finite region. The spatial dimension is explicitly present in their formulation. Somewhat related, Grossglauser and Tse [10] studied the performance of adhoc networks under mobility. They focused on connected networks, where nodes can either communicate directly or via multi-hop connections, and showed that mobility increases the overall capacity in the network given the users tolerate additional delays. 
In this paper, we consider a fundamental feasibility problem of DTN-style communication with the elementary mobility model by means of percolation theory [11], [12]. Percolation theory has been succesfully applied to study the performance of wireless multi-hop networks since the early work by Gilbert [13]. Similarly as in [13], most of the work utilizes undirected planar continuum percolation models to argue, e.g., about the network's connectivity or capacity.

In contrast, in [14], we assumed stationary nodes and showed that opportunistic content dissemination schemes, such as the floating content, can be analyzed by using a threedimensional continuum percolation model. In this paper, we adapt the same approach, but instead of approximating the process by undirected percolation model, we consider the actual directed percolation characterizing the dissemination of messages by mobile nodes exactly in a DTN network. There are less results available for the directed percolation models than there are for the normal undirected percolation, and results for directed continuum models are even more scarce. The basic directed cases are the bond and site percolation models, where, e.g., bonds have fixed directions (e.g., up and right in a square lattice) [11], [15]. Directed models have been also studied in the context of scale-free networks [16], which arise in Internet, social networks etc.

The direction property can be defined many ways. The model considered in this paper, is a specific case of directed continuum percolation, where three-dimensional objects may belong to a given cluster fully or partially. In particular, the third dimension is time and, due to the causality, only the post-contact part of an object belongs to the given cluster. In this setting, we obtain an elegant fundamental result for the minimum feasible mean node degree to support DTN communication as a function of the ratio of movement to the transmission range. This critical percolation threshold is determined by Monte Carlo simulations. The obtained results define the feasible operation regime for large DTN networks.

The rest of the paper is organized as follows. In Section II we introduce the model and the notation. Section III contains the analysis and the description of the Monte Carlo simulations. The numerical results are given in Section [V] and Section $\mathrm{V}$ concludes the paper.

\section{Model AND Notations}

In this section, we describe our model, including the arrival process, nodes' mobility and the assumptions regarding the radio communication. Throughout this paper, we implicitly assume a large network and a long distance, either in space and/or time, between the source and the destination.

1) Node mobility: We assume that nodes arrive according to a Poisson point process on an infinite plane with rate density denoted by $\lambda$ [node $\left./ \mathrm{m}^{2} / \mathrm{s}\right]$. Mobility is specified with a constant movement $\ell$, i.e., during the constant time $t$ a node moves the distance of $\ell$ to a random direction (isotropic). After the movement, the node departs from the system. Note that this activity cycle from "an arrival" to "a departure" can model an activity period of a node (from active state to inactive state),

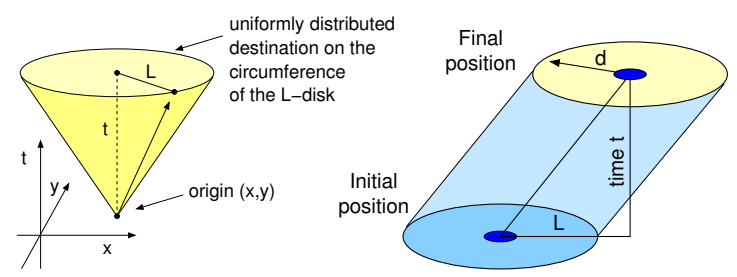

Figure 1: Node mobility in space-time: (left) during time $t$, a node moves a distance of $\ell=L$ to a random direction in $(x, y)$-plane and departs. (right) The volume covered corresponds to a tilted cylinder.

or the departure can correspond to an event where a node replaces the information content with another.

The node density in plane is

$$
\tilde{n} \triangleq \lambda \cdot t,
$$

The arrival-departure cycle can be interpreted as the time when a mobile node is participating in the DTN activity. In the basic stationary case, $\ell=0$ and the nodes remain still for the constant time $t$ before departing. We study the capacity of a large network to transport messages in space-time. Thus, the node mobility model applies to the intermediate nodes, while the source and destination node can be thought to be, e.g., two permanent nodes located far apart from each other.

2) Information dissemination: Whenever two nodes are within each others' transmission range $d$, i.e., the distance between them is less than $d$, they immediately exchange the messages. The assumption of a fixed transmission range is often referred to as the Gilbert's disc model or boolean model [13]. The mean number of neighbors a node has, i.e., the mean node degree is thus $\nu=\tilde{n} \cdot \pi d^{2}$. In the percolation theory, two objects are connected if they overlap and thus the corresponding radius $r$ is half of the transmission range,

$$
r=d / 2 .
$$

3) Space-time: The movement and the radio coverage of a node in space-time, comprising $(x, y)$-plane and the time axis, are depicted in Fig. 1. The radio coverage at any time instant is a disc. As nodes move in time, the coverage in 3D spacetime is a sheared cylinder. The tilt of the cylinder is defined by the ratio of the movement to the transmission range,

$$
\gamma \triangleq \frac{\ell}{d}=\frac{\ell}{2 r} \text {. }
$$

Somewhat counter-intuitively, the number density of nodes (cylinders) in space-time is equal to the arrival rate $\lambda, 1$

$$
n=\lambda \text {. }
$$

Instead of number density, the density of objects is often expressed using either the reduced number density $\eta$,

$$
\eta \triangleq n \cdot V
$$

where $V$ is the volume of the shape, or the volume fraction $\phi$, for which it holds that $\phi=1-e^{-\eta}$.

\footnotetext{
${ }^{1}$ Consider a volume $V=A T$, with area $A$ in $(x, y)$-plane and height $T$. The mean number of nodes in $V$ is $\lambda V$, i.e., the node density $n$ is $\lambda$.
} 
In our case, $V=\pi r^{2} \cdot t$ independently of $\gamma$. Without lack of generality, we can scale the space-time. The time axis can be scaled by constant $\alpha$, e.g., so that $t=1$. Similarly, the $(x, y)$ plane can be scaled by constant $\beta$, e.g., so that $2 \cdot \ell+d=1$. The ratio $\gamma$ is clearly invariant under such scalings. Similarly,

$$
n \propto \alpha^{-1} \beta^{-2} \text { and } V \propto \alpha \beta^{2},
$$

and thus also the reduced number density $\eta=n \cdot V$ and the volume fraction $\phi$ are invariant.

\section{ANALYSIS}

In this section, we first study the corresponding directed continuum percolation model characterizing the evolution of a message in space-time. Then we describe Monte Carlo simulations to estimate the critical percolation threshold, and also derive an asymptotic scaling law for the threshold.

\section{A. Directed continuum percolation}

We consider a dynamic system in space-time, where each node corresponds to a (tilted) cylinder as depicted in Fig. 1 . Two nodes can communicate from the moment the distance between them becomes less than the constant transmission range $d$. It follows that the process describing the evolution of a message in the infinite plane is equivalent to a directed continuum percolation of aligned or sheared cylinders in three dimensions [14]. The radius $r$ of the cylinders with respect to percolation is equal to $d / 2$, and the constant holding time $t$ corresponds to the height of the cylinders. The moment two $r$-cylinders touch each other, messages can be transmitted. The causality imposes that the information flows only in the direction of the positive time axis.

Formally, let $a \rightsquigarrow b$ denote that a path from node $a$ to node $b$ exists in space-time in the sense that $a$ could send a message to $b$ (potentially via a multi-hop connection). Due to the causality, $\rightsquigarrow$ is not a symmetric relation,

$$
a \rightsquigarrow b \quad \nRightarrow \quad b \rightsquigarrow a .
$$

Moreover, $\rightsquigarrow$ is not even transitive,

$$
a \rightsquigarrow b \text { and } b \rightsquigarrow c \quad \nRightarrow \quad a \rightsquigarrow c,
$$

as a message from $a$ to $b$ may arrive too late for $b$ to deliver it to $c$. Let $\mathcal{S}(a)$ denote the set of nodes reachable from $a$,

$$
\mathcal{S}(a)=\{b: a \rightsquigarrow b\} .
$$

We are interested in the size (i.e., the cardinality) of $\mathcal{S}(a)$, and let random variable $S$, cluster size, denote this quantity,

$$
S \triangleq|\mathcal{S}(a)| \text {. }
$$

To illustrate the directed percolation, consider a sample clusters depicted in Fig. 2 obtained without mobility $(\ell=0)$. The time axis points towards the top-right corner and without mobility all cylinders are aligned with the time axis. The dark red node (cylinder) indicates the source of a message, light yellow color indicates that a node has not obtained the message yet, and green color means that the node has it (at the given time). Those nodes that never obtained the message

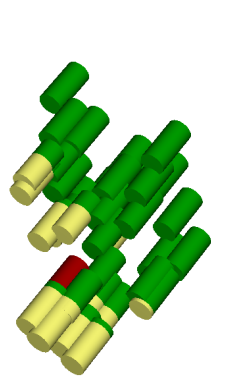

37 nodes

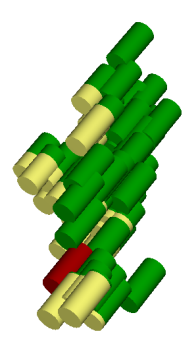

67 nodes

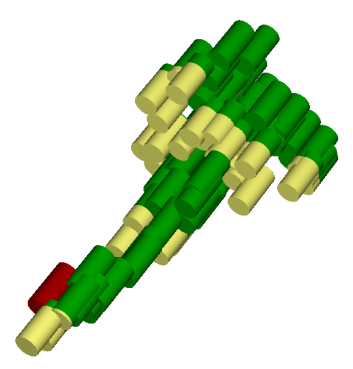

86 nodes
Figure 2: Sample realizations of dissemination of a message with stationary nodes. The reduced number density is $\eta=0.37$. The red cylinder is the source, the light yellow color indicates that a node is waiting for the message and the green color that a node has acquired it. Nodes which did not acquire the message have been omitted.

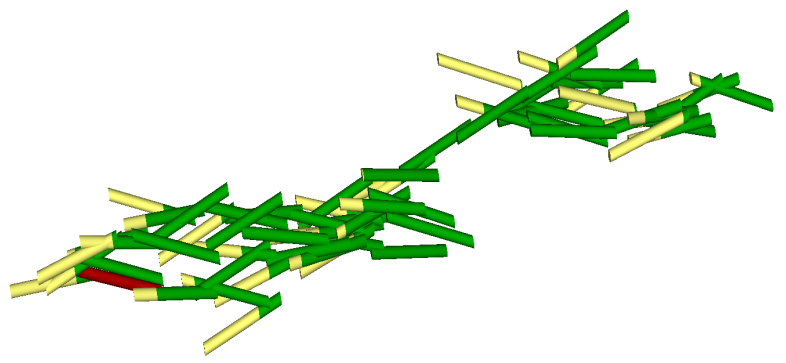

Figure 3: Sample realization with tilt $\gamma=1$ and $\eta=0.14$ comprising 65 nodes.

have been omitted for clarity. Fig. 3 illustrates the situation with mobile nodes. From the figures we observe how the information "flows" only in the positive direction of time.

When the number density of the cylinders (in space-time) is above the critical threshold, denoted by $n_{c}$, there is a positive probability that a random cylinder belongs to an infinite cluster. Mathematically the critical percolation density is defined as,

$$
n_{c} \triangleq \inf \{n \mid \mathrm{P}\{S=\infty\}>0\} .
$$

From the information dissemination point of view, this means that there is a positive chance that a given message gets distributed "everywhere" and does not go into extinction.

The critical number density $n_{c}$ obviously depends on the dimensions of the cylinders and the amount of the movement $\ell$. Therefore, the critical percolation threshold is often expressed using either the critical reduced number density $\eta_{c}$ or the critical volume fraction $\phi_{c}$, which are both invariant to scaling as explained earlier. That is, for the aligned cylinders with $\gamma=0$, it holds that $\eta_{c}$ and $\phi_{c}$ are independent of the dimensions of the cylinder [14]. In our case, the cylinders are tilted to a random direction, and consequently, the criticality threshold depends on the tilt ratio $\gamma$, and we have $\eta_{c}=\eta_{c}(\gamma)$ (i.e., $\gamma$ is a shape parameter). Formally,

$$
\eta_{c}(\gamma) \triangleq \inf \left\{\eta \mid \mathrm{P}\left\{S_{\gamma}=\infty\right\}>0\right\}
$$

Two larger sample clusters are shown in Fig. 4. The cluster 


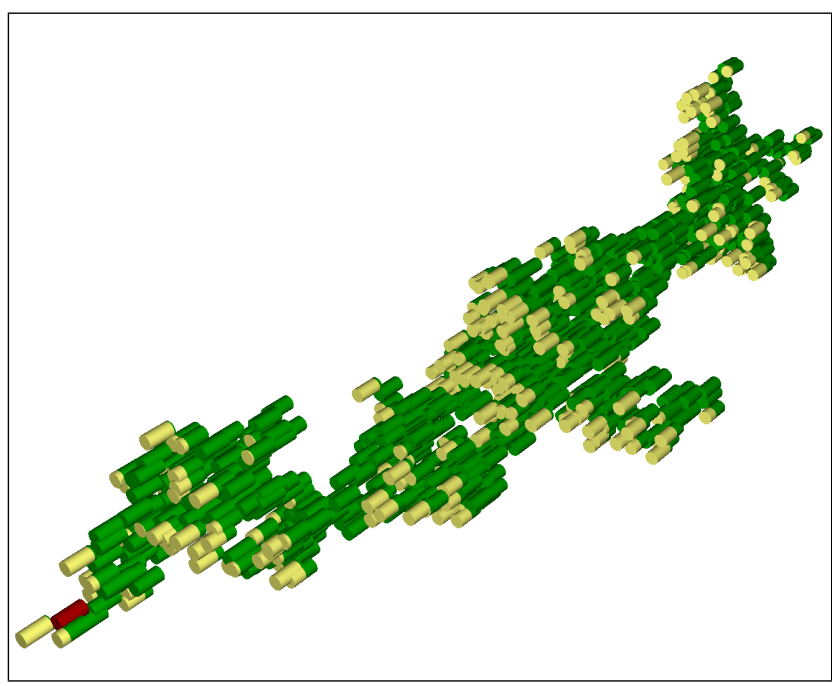

A sample 969 node cluster with $\eta=0.37$.

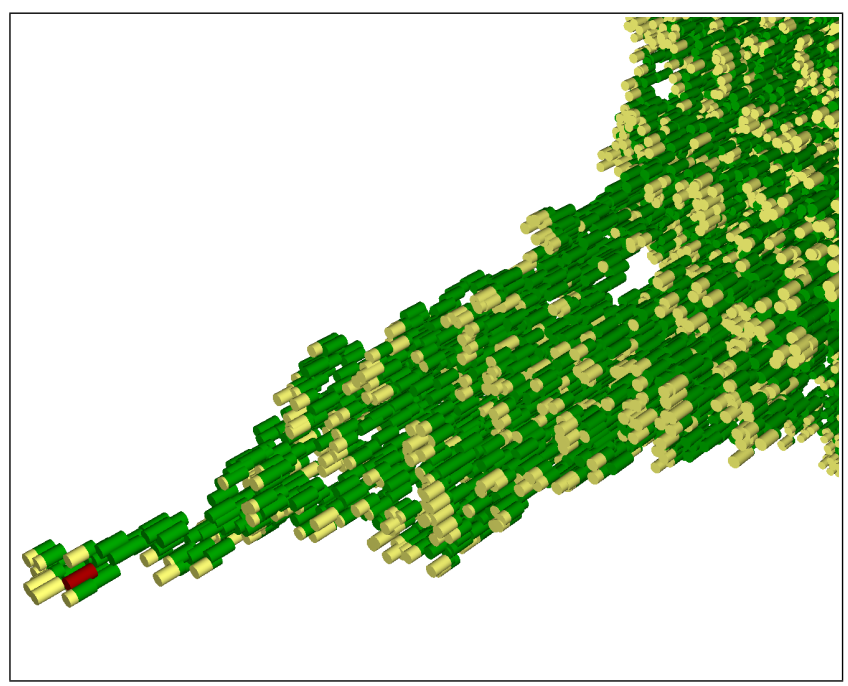

A presumably infinite cluster with $\eta=0.39$.

Figure 4: Sample realizations of a message dissemination with stationary nodes. Left figure illustrates a finite cluster with 969 nodes obtained with $\eta=0.37$. In the right figure, the number density is higher, $\eta=0.39$, which is above the percolation threshold and the sample realization (presumably) percolates.

in left is finite and the message goes to extinction after some time, while the cluster on the right appears to lead to an infinite cluster, i.e., the system percolates implying ${ }^{2} \eta>\eta_{c}$.

The volume of the (tilted) cylinders is $V=\pi r^{2} \cdot t$ and the number density in space-time is $n=\lambda$. Thus, $\eta=\pi r^{2} \cdot \lambda t$, yielding

$$
\nu=4 \eta .
$$

Consequently, in terms of the mean node degree, the criticality condition states

$$
\nu_{c}(\gamma)=4 \eta_{c}(\gamma)
$$

An infinite cluster with respect to the directed percolation is naturally an infinite cluster also with respect to the undirected percolation. In [14], we already determined the critical (undirected) continuum percolation threshold for aligned cylinders, $\eta_{c}^{*}=0.3312(1)$, corresponding to the case $\ell=\gamma=0$, which thus serves as a strict lower bound for the directed percolation in the same setting, $\eta_{c}(0)>\eta_{c}^{*}(0)=0.3312(1)$.

When a DTN network percolates in space-time, it means that there is a positive probability that a message makes its way to the destination no matter how far the destination is (in space). In this sense, the criticality condition (3) is a fundamental result for the transport capacity of DTN. At the same time, it gives no guarantees on the probability that this happens for a particular message (cf. strength of the percolation), or on how long it takes (which obviously depends on the distance among other things, cf. [9]).

\section{B. Methodology}

An elegant way to determine the percolation threshold is based on the asymptotic behavior of the cluster size $S$. In

\footnotetext{
${ }^{2}$ What happens when $\eta=\eta_{c}$ is not known in general, but for $\eta<\eta_{c}$, by definition, no infinite cluster emerges.
}

particular, the tail behaves according to

$$
\mathrm{P}\{S \geq s \mid \eta\} \sim A s^{2-\tau} f\left(\left(\eta-\eta_{c}\right) s^{\sigma}\right),
$$

where $\tau$ and $\sigma$ denote the so-called universal exponents and $A$ is some (non-universal) constant. In three dimensions [17],

$$
\begin{aligned}
\tau & =2.18906 \pm 0.00006 \\
\sigma & =0.4522 \pm 0.0008
\end{aligned}
$$

Near the percolation threshold, where $\eta \approx \eta_{c}$. The Taylor series for $f(x)$ is $f(x)=1+B x+\ldots$, which gives

$$
\mathrm{P}\{S \geq s \mid \eta\} \cdot s^{\tau-2} \sim A+A B\left(\eta-\eta_{c}\right) s^{\sigma}+\ldots,
$$

i.e., the quantity on the left-hand side becomes a constant when $\eta=\eta_{c}$. The critical $\eta_{c}$ is then determined as follows [14], [18]-[20]. First one collects a large sample set of cluster sizes and stores the results to bins so that the $k$ th bin, denoted by $B_{k}$, corresponds to the number of samples with cluster size $s \geq 2^{k}$. For $\eta=\eta_{c}$, the quantity $B_{k} \cdot\left(2^{k}\right)^{\tau-2}$ then has a constant tail. Thus, by trial and error such $\eta$ is determined.

In general, there are fewer results available for the directed percolation than there are for the undirected percolation. For example, the universal exponents (4) may be different. Hence, in Section IV, we investigate the percolation threshold of the directed model first by examining the CDF of the cluster size, and then observe that the universal exponents may well be the same for both cases. To this end, we have developed a fast Monte Carlo simulation tool that provides us with samples from the cluster size distribution. The pseudo code of the core routine is given in Algorithm 1

The algorithm partitions the space-time into unit boxes as illustrated in Fig. 5. The dimensions are chosen in such a way that a node appearing to the shaded box in the center can interact during its lifetime only with nodes for which the initial position was in one of the 27 surrounding boxes including the 


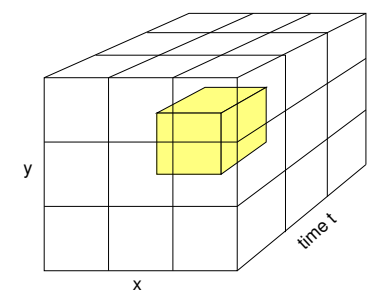

Figure 5: Partitioning of the $3 D$ space-time.

box in the center. This gives a significant reduction in the running time of the algorithm, where for each arriving node one needs to check the contact times with all other nodes. As we may be dealing with infinite clusters, an additional condition for termination is needed. To this end, we have defined the maximum cluster size $s_{\max }$ we are interested in.

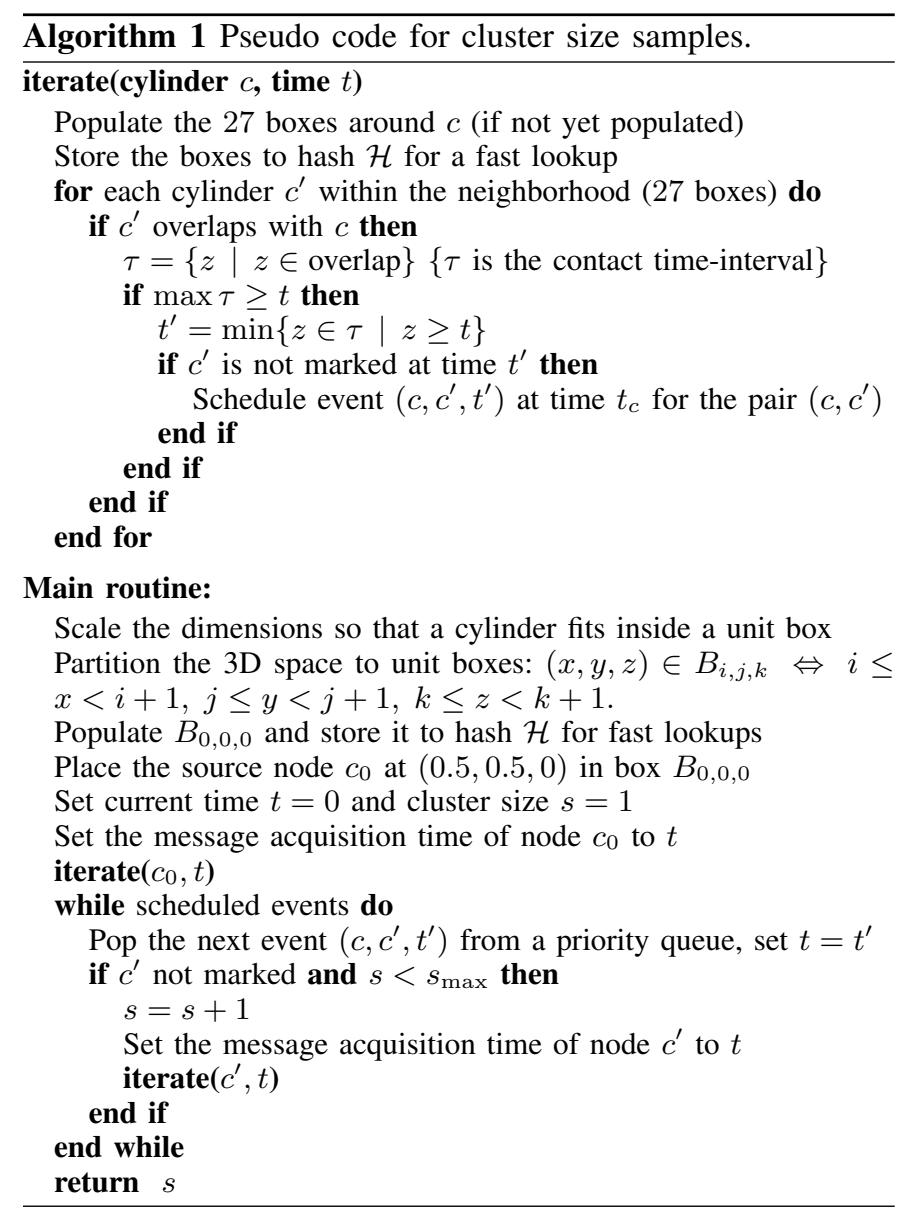

\section{Asymptotic behavior}

Before proceeding with the numerical results according to the percolation theory, let us first discuss the asymptotic behavior when the distance $\ell$ is much longer than the transmission range $d, \ell \gg d$. In this case, the situation is similar to the one analyzed in [2], [21]: contacts with other nodes are point contacts, which occur at a constant rate of

$$
\tilde{\lambda} \triangleq \frac{8}{\pi} \tilde{n} d
$$

per unit distance on the $(x, y)$-plane. Near the criticality threshold, only a small fraction of nodes carry the message. Assuming a node meets at most one node with the message, the contact time is uniformly distributed on interval $(0, \ell)$ along the path. In order for the system to avoid extinction, each node acquiring the message should pass it further to at least one other node on average. That is,

$$
\frac{1}{\ell} \int_{0}^{\ell} s \frac{8}{\pi} \tilde{n} d d s>1
$$

giving the fluid bound (fb),

$$
\nu>\nu_{c}^{(\mathrm{fb})}(\gamma) \triangleq \frac{\pi^{2}}{4 \gamma},
$$

which is valid when $\gamma$ tends to infinity. At this bound, $\tilde{\lambda} \cdot \ell=2$, i.e., a node meets on average only two nodes before departing. Conversely, with aid of (2), we find a scaling law for the tail of $\eta_{c}(\gamma)$,

$$
\eta_{c}(\gamma) \propto 1 / \gamma, \quad \text { for } \gamma \gg 1
$$

It is straightforward to generalize the above to non-constant transitions, i.e., to the case where the movement $\ell$ is an i.i.d. random variable, $\ell \sim X$, with $\operatorname{PDF} f(x)$. The point contact assumption means that the transitions must be (almost surely) such that $\ell \gg d$. Moreover, in a microscopic scale the movement is assumed to be isotropic and direct, while in a macroscopic scale the paths can make, e.g., smooth turns [21]. The criticality condition is obtained as in above: each node acquiring a message should, on average, pass it to one new node. According to the theory of renewal processes (see, e.g., [22]), the PDF for transition length $\ell$ on condition that a node obtains a message is

$$
g(x)=x f(x) / \mathrm{E}[X]
$$

(cf. the hitchhiker's paradox). The remaining transition length $R$ after the acquisition on condition that $\ell=x$ is uniformly distributed on $(0, x)$ and $\mathrm{E}[R]=x / 2$. The number of new contacts $A$ in $R$ is

$$
\mathrm{E}[A \mid \ell=x]=\frac{4}{\pi} \tilde{n} d x .
$$

Hence, the mean number of contacts after the acquisition is

$$
\mathrm{E}[A]=\int_{0}^{\infty} g(x) \mathrm{E}[A \mid x] d x,
$$

and requiring $\mathrm{E}[A]>1$ then yields,

$$
\tilde{n} d>\frac{\pi \mathrm{E}[X]}{4 \mathrm{E}\left[X^{2}\right]} .
$$

The second moment in the denominator underlines the fact that varying transition length improves the transport capacity in DTN communication. 

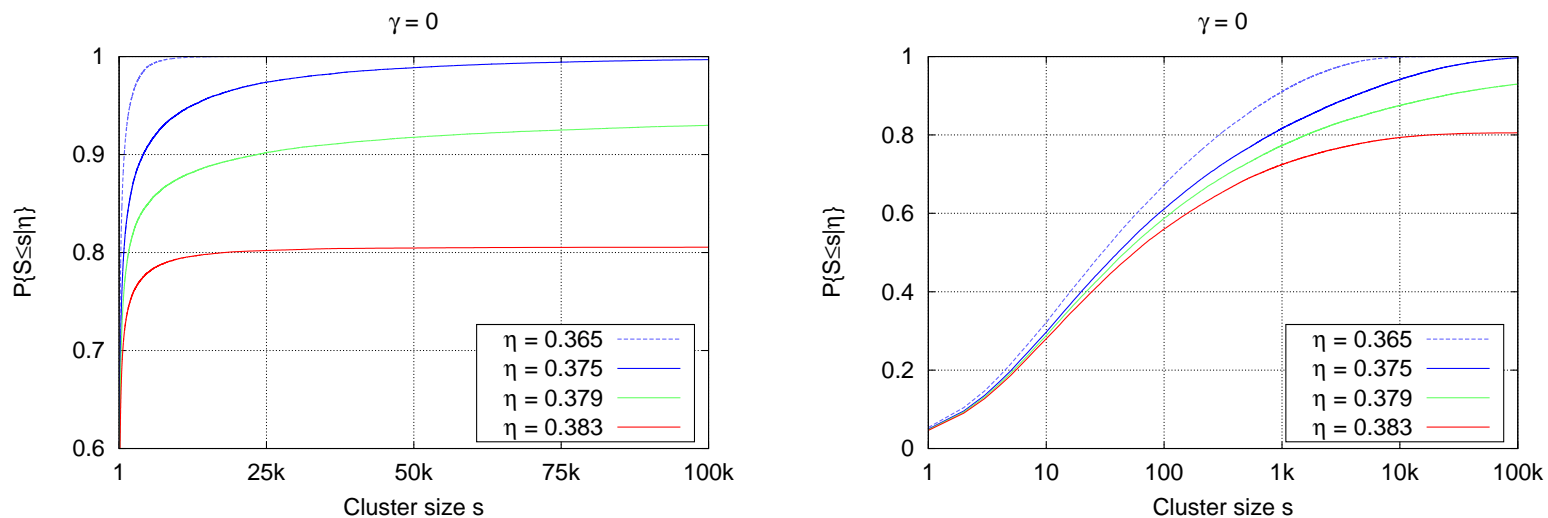

Figure 6: Numerical results with stationary nodes, $\gamma=0$. Note the linear scale on the left figure and logarithmic scale on the right figure.
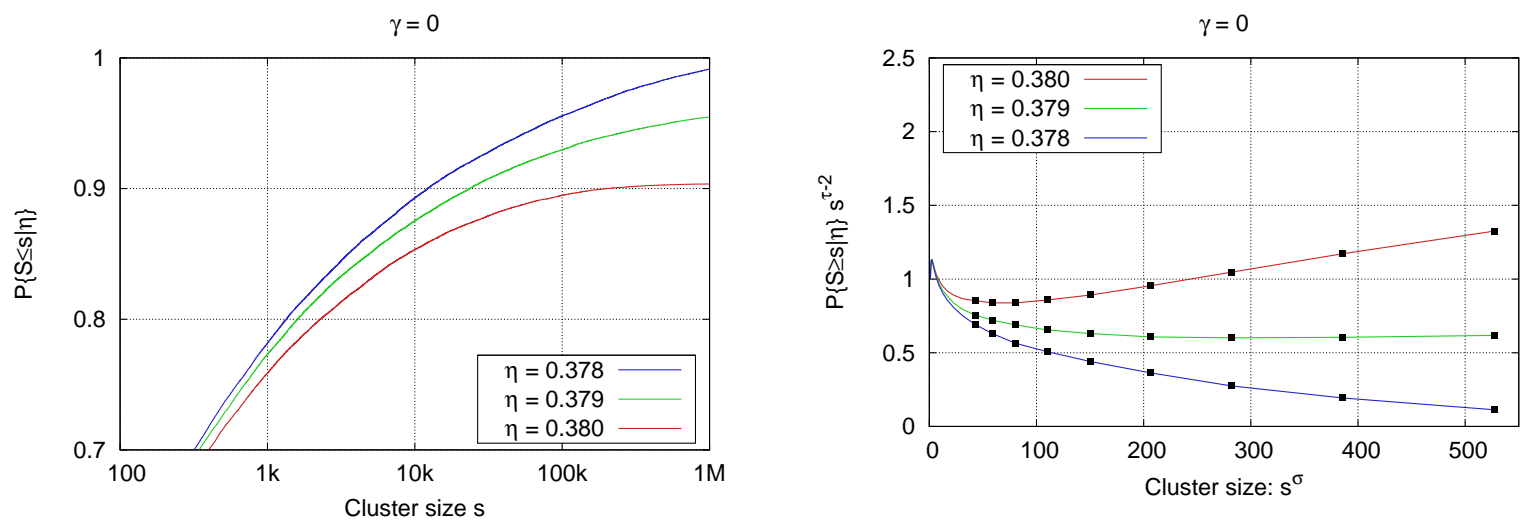

Figure 7: Closer look at the numerical results with stationary nodes, $\gamma=0$.

\section{NUMERICAL RESULTS}

We have carried out a large number of Monte Carlo simulations with the aforementioned simulation tool in order to determine numerical values for $\eta_{c}(\gamma)$. The sample size for each $(\eta, \gamma)$-pair is 60000 clusters. The parameter $s_{\max }$, defining the maximum cluster size, was chosen to be $2^{20}+1=1048577$, i.e., about 1 million. With these values, the running time is still reasonably short with a standard PC. Such clusters are rather large creatures, e.g., there are more than $10^{12}$ node pairs, and a naïve implementation would choke on them.

\section{A. Stationary nodes}

First we assume no mobility, i.e., $\gamma=0$, and vary the reduced number density, $\eta=0.365, \ldots, 0.383$, which corresponds to the mean node degree of $\nu=1.46, \ldots, 1.53$. If $\nu \approx 4.51$ or higher, Gilbert's disc model percolates and a gigantic component emerges (at any given time instant) enabling multi-hop communication [1]. However, in our case the node density is clearly below that and one has to resort to DTN-style communication. This is illustrated in Fig. 8, where $\nu=1.52$ in the left figure and $\nu=4.51$ in the right figure.

Recall that the percolation threshold $\eta_{c}$ corresponds to the smallest node density at which a gigantic component emerges,

$$
\eta_{c}=\inf \{\eta \mid \mathrm{P}\{S=\infty\}>0\} .
$$

Therefore, if CDF of the cluster size $S$ remains asymptotically below $1, \lim _{s \rightarrow \infty} \mathrm{P}\{S<s\}=p$ and $p<1$, then the given system is above the percolation threshold. The quantity $1-p$, referred to as the strength, is the probability that a cluster starting from a random node is infinite [11].

The simulation results are illustrated in Fig. 6. The left figure depicts CDF with a linear scale on the $x$-axis, and the right figure with a logarithmic scale. The logarithmic scale gives more insight to the behavior. The CDF for $\eta=0.365$ converges to 1 at $s \approx 10^{4}$, i.e., in practice every realization goes to extinction and no message will be delivered for more than to about $10^{4}$ nodes. However, the CDF for $\eta=0.379$ seems to converge to a finite value less than 1 , suggesting that a small fraction of messages may survive forever. Increasing $\eta$ further to 0.383 improves the chances of hitting a gigantic cluster to about $20 \%$, i.e., with $\eta=0.383$ the strength of the percolation is about 0.2 .

Conservatively, we observe that $\eta=0.383$ is above the percolation threshold, while $\eta=0.375$ is most likely below it, $0.375<\eta_{c}<0.383$. Comparing the directed percolation to the undirected one, we see that the direction constraint causes $\eta_{c}$ to increase from $0.3312(1)$ to about 0.38 , i.e., about $15 \%$ increment. This is in agreement with the observations made in [14], where an actual content dissemination system (obeying the causality) was also evaluated by means of simulations. 

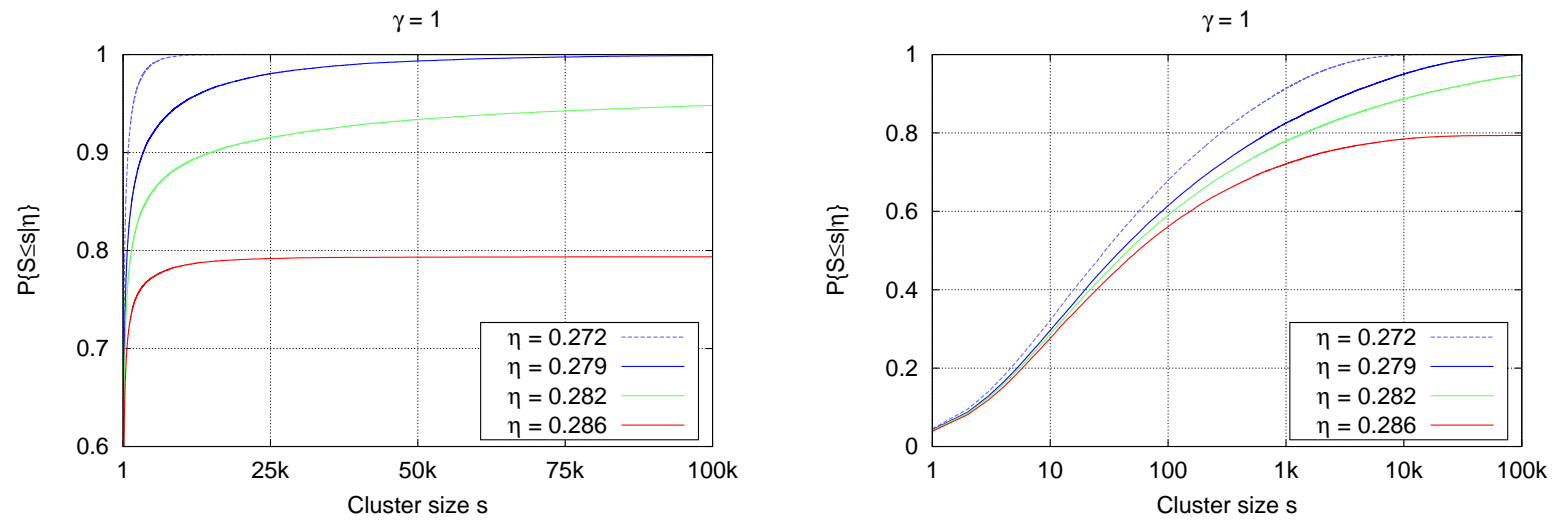

Figure 9: Numerical results with mobile nodes, $\gamma=1$.
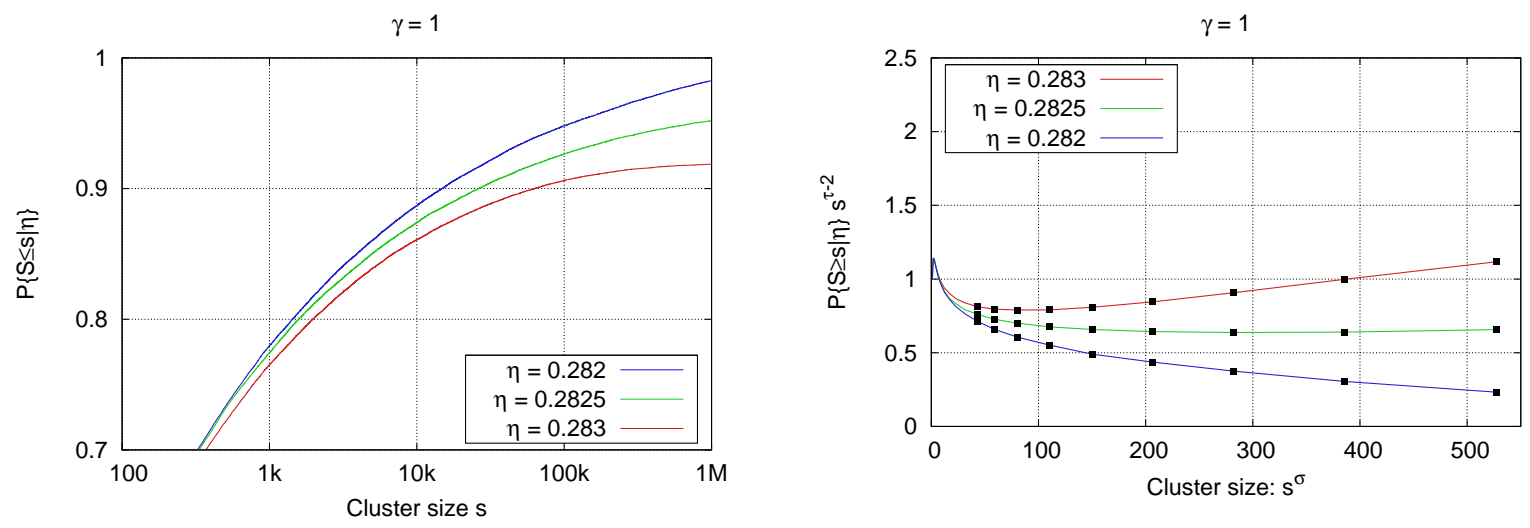

Figure 10: Closer look at the results with mobile nodes, $\gamma=1$.

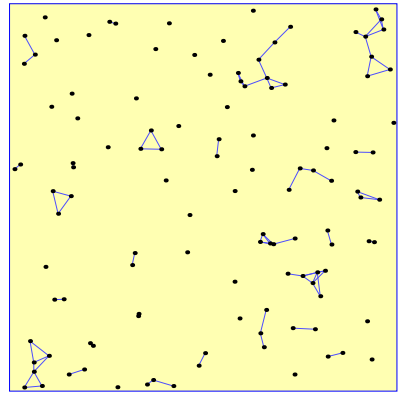

$\nu=1.52$

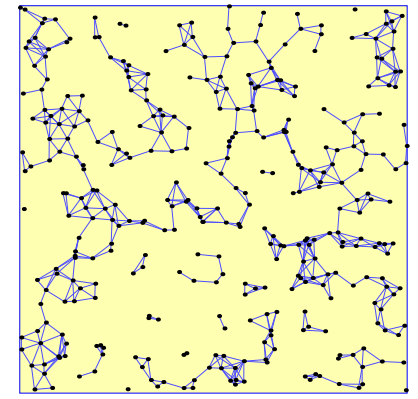

$\nu=4.51$
Figure 8: Snapshot of two networks.

1) Universal exponents: As mentioned, it is not clear whether the universal exponents (4) are the same for the directed model and the normal undirected model. Fig. 7 (left) shows CDF of the cluster size distribution in the interesting region near the percolation threshold. The graph clearly suggests that $0.378<\eta_{c}(0)<0.380$ and our estimate is $\eta_{c}(0) \approx 0.379$. In Fig. 7 (right), the universal exponents according to (4) are used. The curve corresponding to $\eta=0.379$ has a constant tail, which suggests that the universal exponents are the same for the undirected and directed percolation models, and we assume so in the rest of the paper. (cf. also the Appendix).

\section{B. Mobile nodes}

Next we set parameter $\gamma$ to 1 , i.e., each node moves a distance equal to the transmission range before departing. The numerical results are given in Fig. 9, where the left figure is again in linear scale and the right in logarithmic. We observe that the critical $\eta_{c}(1) \approx 0.282$, as the curve with $\eta=0.279$ clearly converges to 1 and the curve with $\eta=0.286$ stabilizes at about 0.8. Fig. 10 takes a closer look at the interesting regime. Left figure depicts the CDF, which suggests that $0.282<\eta_{c}(1)<0.283$. In the right figure, we again use the universal exponents and the curve with $\eta=0.2825$ indeed appears to have a constant tail. However, a closer inspection of the curve reveals that in fact it is slightly increasing already at $s^{\sigma}=500$, suggesting $\eta_{c}<0.2825$. As three digits is more than enough for our purposes, we conclude that $\eta_{c}(1) \approx 0.282$.

Recall that $\nu=4 \eta$ according to (2), i.e., the minimum feasible mean node degree $\nu_{c}(\gamma)$ is obtained by multiplying the corresponding $\eta_{c}(\gamma)$ by 4 . According to our numerical results, $\eta_{c}(1) \approx 0.75 \cdot \eta_{c}(0)$, i.e., $25 \%$ smaller. This means that both the node density $n$ and the mean node degree $\nu$ can also be $25 \%$ smaller, i.e., even a small mobility equal to the transmission range improves the message forwarding capacity considerably (in space-time).

The critical reduced number density $\eta_{c}(\gamma)$ for several other values of $\gamma$ is determined in the Appendix. Fig. 11 depicts the 


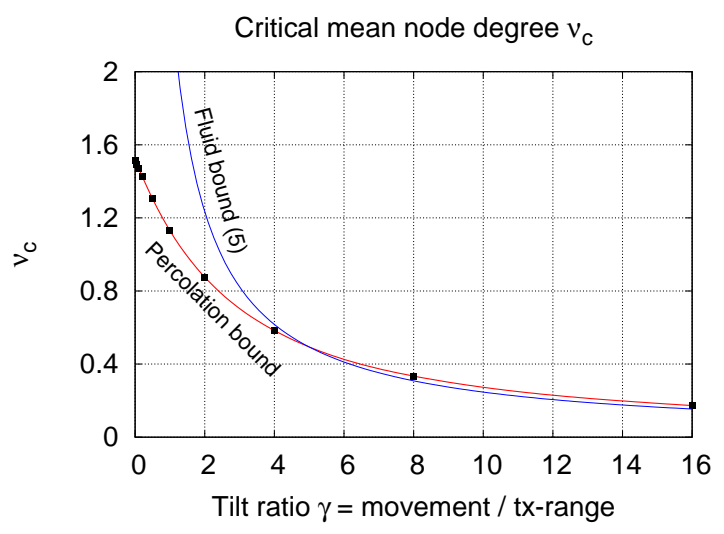

Figure 11: Critical mean node degree $\nu_{c}(\gamma)$ as a function of the tilt ratio $\gamma$ characterizing the relative mobility in the model. The fluid bound curve is according to (5) and valid at the limit $\gamma \rightarrow \infty$. The other curve is due to percolation theory, $\nu_{c}(\gamma)=4 \eta_{c}(\gamma)$.

behavior of the critical mean node degree $\nu_{c}(\gamma)$ as a function of $\gamma$. The fluid bound curve is according to (5) and valid at the limit $\gamma \rightarrow \infty$. The other curve corresponds to the critical percolation threshold, $\nu_{c}(\gamma)=4 \eta_{c}(\gamma)$, the values of which we have obtained numerically in this paper. We observe that the highest gain from an additional movement of $\Delta \ell$ is obtained when $\gamma=0$, as expected.

The two curves differ initially, where (5) is not valid. However, already at $\gamma \approx 4$ the two curves are almost matching. Consequently, for a small movement one needs to analyze the situation according to the percolation, but as soon as, say, $\gamma \geq 4$, the fluid flow analysis already gives accurate results.

\section{CONCLUSIONS}

DTN is designed to operate in settings where connectivity is intermittent at best. In this paper, we have analyzed necessary conditions for DTN type communication. To this end, we assumed an elementary mobility model where nodes join the network for a constant time $t$ and then depart. During the sojourn time, they move a distance $\ell$ to a random direction. We assume a large network, where the ability to sustain a message indefinitely means that the message will eventually also reach its final destination(s). The stochastic model of DTN is amenable to analysis by means of the percolation theory. In particular, we studied the so-called directed continuum percolation in space-time, where the objects are cylinders with height $t$ and the diameter equal to the transmission range $d$ sheared (tilted) around the time axis by an amount corresponding to the movement $\ell$. We showed that the critical percolation threshold in terms of the critical reduced number density $\eta_{c}$ depends only on the tilt ratio $\gamma=\ell / d, \eta_{c}=\eta_{c}(\gamma)$, where $\ell$ denotes the distance nodes move and $d$ is the transmission range. Numerical values for $\eta_{c}(\gamma)$ were obtained by means of Monte Carlo simulations, where universal exponents of the undirected model were utilized. Some evidence to support the hypothesis that the universal exponents are the same for the directed model was also given. Moreover, the asymptotic behavior of $\eta_{c}(\gamma)$ when $\gamma$ tends to infinity was derived. In terms of the mean node degree $\nu$, our main result states that $\nu>4 \eta_{c}(\gamma)$ in order for a large DTN network to be operational. A general observation is that increased mobility improves the transport capacity of DTN and allows a lower node density, as expected.

The elementary mobility model, as well as, the symmetric Gilbert's disc communication model, represent, in some sense, a strict lower bound for more realistic scenarios. That is, if, e.g., the sojourn time $t$ or the distance $\ell$ were random variables, or, if directional antennas were used, then the shapes and the orientation of the objects would vary more, increasing "disorder" and yielding a lower critical percolation threshold. Such studies are left as a future work.

\section{REFERENCES}

[1] M. Franceschetti, O. Dousse, D. Tse, and P. Thiran, "Closing the gap in the capacity of wireless networks via percolation theory," IEEE Transactions on Information Theory, vol. 53, no. 3, Mar. 2007.

[2] E. Hyytiä, J. Virtamo, P. Lassila, J. Kangasharju, and J. Ott, "When does content float? characterizing availability of anchored information in opportunistic content sharing," in IEEE INFOCOM, Shanghai, China, Apr. 2011, pp. 3123-3131.

[3] J. Ott, A. Keränen, and E. Hyytiä, "Beachnet: Propagation-based information sharing in mostly static networks," in ExtremeCom 2011 - The Amazon Expedition, Manaus, Brazil, Sep. 2011.

[4] A. Villalba Castro, G. Di Marzo Serugendo, and D. Konstantas, "Hovering information: Self-organizing information that finds its own storage," School of Computer Science and Information Systems, Birkbeck College, London, UK, Tech. Rep. BBKCS707, Nov. 2007.

[5] V. Lenders, M. May, G. Karlsson, and C. Wacha, "Wireless ad hoc podcasting," ACM/SIGMOBILE Mobile Computing and Communications Review, Jan. 2008.

[6] A. Vahdat and D. Becker, "Epidemic routing for partially connected ad hoc networks," Duke University, Technical Report CS-200006, Apr. 2000.

[7] R. Groenevelt, P. Nain, and G. Koole, "The message delay in mobile ad hoc networks," Performance Evaluation, vol. 62, no. 1-4, pp. 210-228, Oct. 2005.

[8] X. Zhang, G. Neglia, J. Kurose, and D. Towsley, "Performance modeling of epidemic routing," Elsevier Computer Networks, vol. 51, pp. 28592891, 2007.

[9] P. Jacquet, B. Mans, and G. Rodolakis, "Information propagation speed in mobile and delay tolerant networks," IEEE Transactions on Information Theory, vol. 56, no. 10, pp. 5001-5015, Oct. 2010.

[10] M. Grossglauser and D. N. C. Tse, "Mobility increases the capacity of ad hoc wireless networks," IEEE/ACM Trans. Networking, vol. 10, no. 4, Aug. 2002.

[11] D. Stauffer and A. Aharony, Introduction To Percolation Theory, 2nd ed. CRC Press, 1994.

[12] R. Meester and R. Roy, Continuum Percolation. Cambridge University Press, 1996.

[13] E. N. Gilbert, "Random plane networks," Journal of the Society for Industrial and Applied Mathematics, vol. 9, no. 4, pp. 533-543, 1961.

[14] E. Hyytiä, J. Virtamo, P. Lassila, and J. Ott, "Continuum percolation threshold for permeable aligned cylinders and opportunistic networking," IEEE Communications Letters, 2012, to appear.

[15] J. A. M. S. Duarte, "Directed lattices: site-to-bond conversion and its uses for the percolation and dilute polymer transitions," Zeitschrift für Physik B Condensed Matter, vol. 80, pp. 299-304, 1990.

[16] N. Schwartz, R. Cohen, D. Ben-Avraham, A. L. Barabási, and S. Havlin, "Percolation in directed scale-free networks," Phys. Rev. E, vol. 66, no. 1, p. 015104, Jul. 2002.

[17] H. G. Ballesteros, L. A. Fernández, V. Martín-Mayor, A. M. Sudupe, G. Parisi, and J. J. Ruiz-Lorenzo, "Scaling corrections: site percolation and ising model in three dimensions," Journal of Physics A: Mathematical and General, vol. 32, no. 1, pp. 1-13, 1999.

[18] P. L. Leath, "Cluster size and boundary distribution near percolation threshold," Phys. Rev. B, vol. 14, pp. 5046-5055, Dec. 1976. 

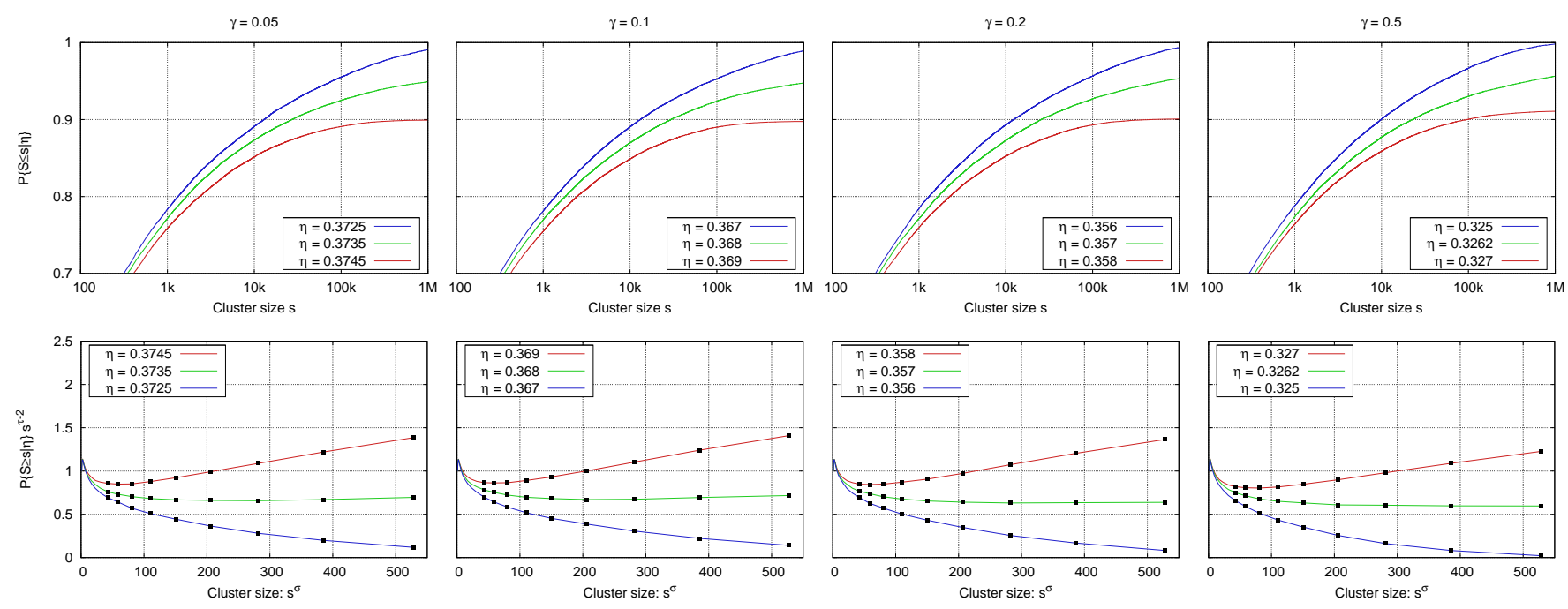

Figure 12: Numerical results for $\eta_{c}(\gamma)$ with $\gamma=0.05,0.1,0.2,0.5$.
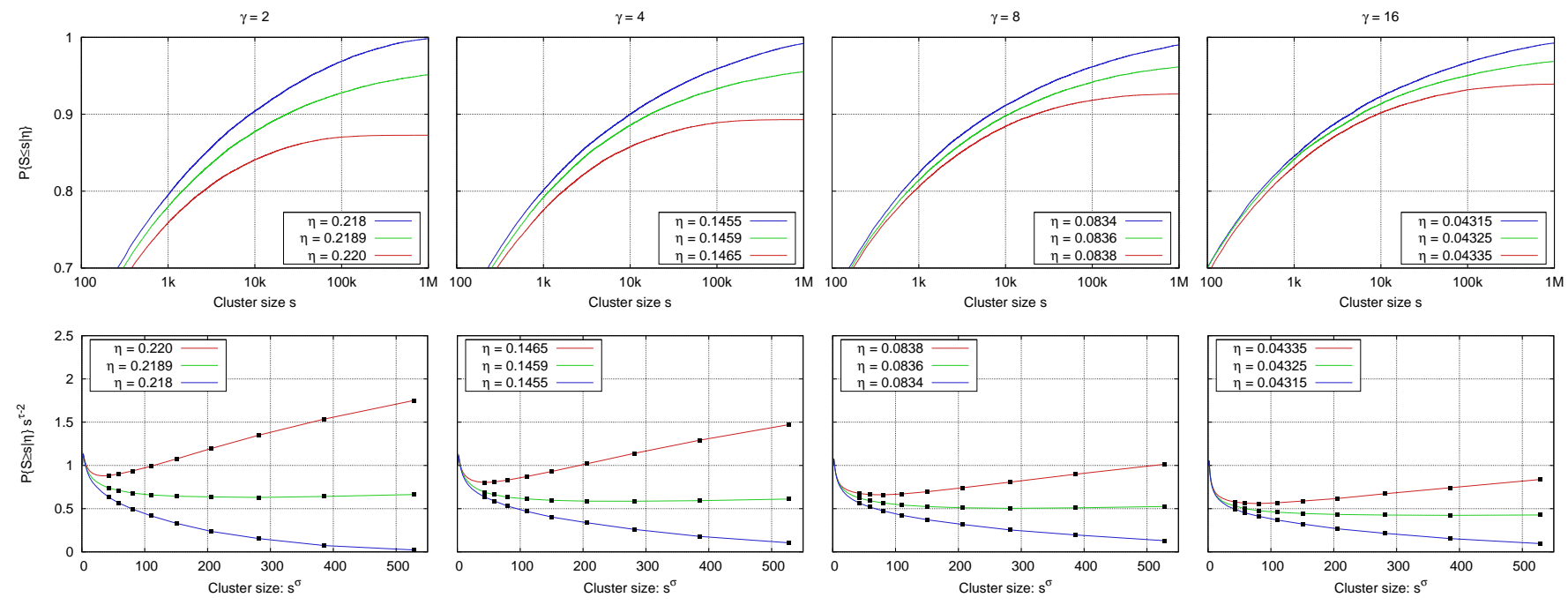

Figure 13: Numerical results for $\eta_{c}(\gamma)$ with $\gamma=2,4,8,16$.

[19] C. D. Lorenz and R. M. Ziff, "Precise determination of the critical percolation threshold for the three-dimensional "swiss cheese" model using a growth algorithm," J. Chem. Phys., vol. 114, 2001.

[20] D. R. Baker, G. Paul, S. Sreenivasan, and H. E. Stanley, "Continuum percolation threshold for interpenetrating squares and cubes," Physical Review E, vol. 66, 2002.

[21] J. Virtamo, E. Hyytiä, and P. Lassila, "Criticality condition for information floating with random walk nodes," Sep. 2012, submitted.

[22] L. Kleinrock, Queueing Systems, Volume I: Theory. Wiley Interscience, 1975.

\section{APPENDIX}

An estimate for the critical reduced number density $\eta_{c}(\gamma)$ is determined for $\gamma=0.05,0.1,0.2,0.5,2,4,8,16$ in Figs. 12 . 13 In each case, parameter $\eta$ obtains values from both sides of the corresponding critical percolation threshold. Table I summarizes the results. In Fig. 14, the maximum cluster size is very large, $s_{\max }=10^{8}$, suggesting $\eta_{c}(0)=0.3788(1)$ and also supporting the hypothesis on the universal exponents.

\begin{tabular}{|ll|rl|}
\hline$\gamma$ & $\eta_{c}(\gamma)$ & $\gamma$ & $\eta_{c}(\gamma)$ \\
\hline 0.0 & 0.3788 & 1 & 0.2825 \\
0.05 & 0.3735 & 2 & 0.2189 \\
0.1 & 0.368 & 4 & 0.1459 \\
0.2 & 0.357 & 8 & 0.0836 \\
0.5 & 0.3262 & 16 & 0.04325 \\
\hline
\end{tabular}

Table I: Critical reduced number density $\eta_{c}(\gamma)$ based on the Monte Carlo simulations, where $\eta_{c}(8) \approx 2 \eta_{c}(16)$ in agreement with 6 .
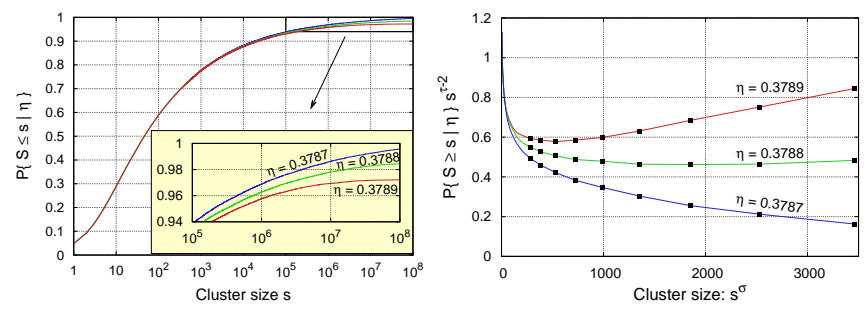

Figure 14: Results with $\gamma=0$ and $s_{\max }=10^{8}$, i.e., 100 million. 